American Journal of Applied Sciences 6 (5): 862-868, 2009

ISSN 1546-9239

(C) 2009 Science Publications

\title{
Somatic Chromosomes of the Bornean Sambar Deer and Rusa Deer Interspecific Hybrids
}

\author{
${ }^{1}$ Ismail Idris and ${ }^{2}$ Saidi Moin \\ ${ }^{1}$ Department of Animal Science, \\ ${ }^{2}$ Department of Biomedical Sciences, \\ University Putra Malaysia, 43400 UPM Serdang, Selangor, Malaysia
}

\begin{abstract}
Problem statement: Hybridization has potential benefits to the Malaysian farmed deer industries in terms of increased growth rate and increased proportion of muscle and an improved alignment of feed supply and annual energy requirement. Species or subspecies of different chromosome constitution could mate to produce healthy hybrid offspring in a normal ratio of males and females. If any of the hybrid offspring were sterile, the sterile offspring would be the heterogametic offspring. The study investigated the use of chromosome banding method to detect chromosomal variation and to define the chromosome homology and the possibility of the Bornean Sambar deer (Cervus unicolor brookei) and Rusa deer (Cervus timorensis) hybrids to reproduce. Approach: Samples were collected from the Livestock Breeding Station, Sabrang, Keningau, Sabah, East Malaysia. The animals studied consisted of two deer subspecies namely the Bornean Sambar deer, Rusa deer and their hybrids. The karyotypes of the Bornean Sambar deer, Rusa deer and their F1 hybrids have been investigated by solid giemsa staining, G-banding and Ag-NOR banding techniques. Results: Rusa and Bornean Sambar have different chromosome number; 60 and 62 respectively, but share the same fundamental number of chromosome arm, 70. The hybrids have $2 n=61$, consisting of 9 metacentric to submetacentric autosomes and 24 pairs of acrocentric autosomes with two acrocentrics and one submetacentric chromosome being unpaired. The morphology of the sex chromosomes in the F1 hybrids was similar to that of the parental species. The Ag-NOR pattern and the conventional Giemsa staining of chromosomes were effective as markers in the characterization of the karyotypes of the parental lines and hybrids because of the presence of active NORs on different chromosomes of different species. G-band, in contrast, showed complete homology in the presence of euchromatic bands and heterochromatin blocks respectively on each chromosome, suggesting that the two species have few genetic differences. Conclusion: In the present study, natural mating between Bornean Sambar deer and Rusa deer were conducted and the number and chromosomal location of the nucleolar organizer regions in their offsprings were analyzed by the silver staining method. Apart from that, the interspecific dissimilarities with regards to chromosome number and morphology are less extensive and the production of chromosomally balanced gametes could be expected.
\end{abstract}

Key words: Hybridization, chromosomes, karyotype

\section{INTRODUCTION}

Interspecies hybridization has potential benefits to the livestock industry in terms of increased growth rate and increased proportion of muscle, extended breeding season and an improved alignment of feed supply and annual energy requirement. Most of the successful interspecies hybrids reported resulted from the presence of genetic compatibility between parental species. Parental species differing cytogenetically in chromosome constitution could produce fertile hybrids.
However, this is not always the case, as some adult hybrids fail to reproduce. In this case, the sterility could be due to the absence of homology between the parental species $^{[1]}$. Study on interspecies hybridization of Bos frontalis and Bos indicus provide cytogenetic information on the mechanisms of the male hybrid infertility and could facilitate the establishment of a stable crossbreed ${ }^{[2]}$. It was shown that compared with conventional cattle the Bos frontalis has a homozygous, species specific $2 / 27$ centric fusion which reduced the diploid chromosome number from 60-58. This provided

Corresponding Author: Ismail Idris, Department of Animal Science, Faculty of Agriculture, University Putra Malaysia, 43400 Serdang, Selangor, Malaysia Tel: (603) 89466895 Fax: (603) 89432954 
further proof that Robertson translocation-type rearrangements have been the major source of interspecies karyotype differences in the evolution of the Bovidae. There was also a report on the cytogenetics of twin offsprings from an interspecies cross in marmosets (Callitrichinae, Platyrrhini), resulting from a pairing between a female Common marmoset (Callithrix jacchus, $2 \mathrm{n}=46$ ) and a male Pygmy marmoset (Cebuella pygmaea, $2 \mathrm{n}=44$ ). Both hybrid individuals had a karyotype with a diploid chromosome number of $2 n=45$. These genomic imbalances were confined to centromeric and telomeric heterochromatin, while euchromatic chromosome regions appeared balanced in all species investigated ${ }^{[3]}$.

Hybridization between deer of the same genus has frequently been recorded among deer populations. Examples include successful hybridization between red deer and wapiti ${ }^{[4]}$, red deer and sambar deer ${ }^{[5]}$ and sika deer crossed with red deer ${ }^{[6]}$. Preliminary study have shown that the Bornean sambar deer ( $C$. unicolor brookei) has $2 \mathrm{n}=62$ and the rusa deer (C. timorensis timorensis) has $2 \mathrm{n}=60^{[7]}$. However the chromosome constitution and banded karyotype of these two subspecies were not extensively studied. G-banded karyotype and location of active ribosomal DNA at the Nucleolar Organizer Regions (NORs) may contribute to the investigation on the homology between parental species and the $\mathrm{F}_{1}$ hybrids. If the parental species differ relatively little genetically, both parental sets of NORs set and G-band patterns will usually be expressed in the $\mathrm{F}_{1}$ hybrids.

The objectives of this study are to define the chromosome homology of the hybrid animals with the parental species and to describe the possibility of the Bornean sambar deer $\mathrm{x}$ rusa deer hybrids to reproducing the parental G-banding and NORs- banding patterns in adult $\mathrm{F}_{1}$ hybrids.

\section{MATERIALS AND METHODS}

The deer were grouped according to their species and raised in separate paddocks grown with guinea grass (Panicum maximum) at the Livestock Breeding Station, Sabrang, Keningau, Sabah in East Malaysia. Supplemental feeding of cattle pellets was given at the rate of one $\mathrm{kg}$ per head per day, with mineral blocks given free choice. At this centre, natural mating is being practiced. The animals were allowed to wallow during the day, in puddles within the paddocks.

Blood samples were collected aseptically from the jugular veins of adult Bornean Sambar deer, C. unicolor brookei (1今̄, 2ㅇ), Rusa deer, C. timorensis timorensis $(1 \hat{\jmath}, 4+)$ and the $\mathrm{F}_{1}$ hybrids $(7 \hat{\jmath}, 2 q)$. Chromosome preparations were made from cultures according to previous study ${ }^{[8]}$, with some modifications. Cultures were set up and grown in a medium containing RPMI $1640\left(\mathrm{Gibco}^{\circledR}\right)$ medium supplemented with $15 \%$ (w/v) newborn calf serum, $1 \%$ penicillin-streptomycin and $1 \%$ pokeweed mitogen $\left(\mathrm{Gibco}^{\circledR}\right)$. The cultures were incubated for $72 \mathrm{~h}$ at $37^{\circ} \mathrm{C}$. Colcemid $\left(\mathrm{Gibco}^{\circledR}\right)$ was added to the cultures $1 \mathrm{~h}$ prior to harvesting. The cells were swelled in hypotonic solution $(0.075 \mathrm{M} \mathrm{KCl})$ for $20 \mathrm{~min}$ at $37^{\circ} \mathrm{C}$. Cells were prefixed three times in Carnoy's solution (3:1 methanol-acetic acid). Air dried slides were prepared.

Silver staining: The slides were pretreated for $15 \mathrm{~min}$ with Borate buffer ( $\mathrm{pH}$ 9.0), rinsed thoroughly in deionized water and air-dried. The aqueous solution of silver nitrate $(50 \% \mathrm{w} / \mathrm{v})$ was applied on the slides and the slides were covered with cover slips, adapted in moist chamber and incubated at $37^{\circ} \mathrm{C}$ for $18 \mathrm{~h}$. Data were analysed using SPSS software.

G-banding: The G-band patterns were obtained following a modification of the previous study ${ }^{[9]}$. Fiveday old slides were treated with $0.25 \%$ trypsin in $\mathrm{pH}$ 6.8 phosphate buffered saline (without magnesium and calcium) for $1 \mathrm{~min}$, rinsed in normal saline and stained with $10 \%$ Giemsa for $5 \mathrm{~min}$. Chromosomes were arranged using a Karyo® software version 3.0.

\section{RESULTS}

Conventional banding: Chromosome counts taken from 20 cells per individual confirmed the diploid number previously described for Bornean sambar deer $(2 \mathrm{n}=62, \mathrm{NF}=70)$ and rusa deer $(2 \mathrm{n}=60, \mathrm{NF}=70)^{[7]}$. The chromosome complement of all individuals contained 4 pairs of submetacentric to metacentric autosomes. In addition, the rusa had one large submetacentric pair. The remaining chromosomes in the complement were acrocentric, thus the differences in diploid number between Bornean sambar deer and rusa deer were attributable to the presence or absence of the biarmed chromosomes. For both species, the $\mathrm{X}$ chromosome was a large acrocentric and the $\mathrm{Y}$ chromosome was a small submetacentric.

The cross between Bornean sambar deer and rusa deer produced $F_{1}$ hybrids with an uneven chromosome number of $2 n=61$. Thirty chromosomes clearly came from the rusa and 31 chromosomes from the Bornean sambar deer. Figure 1a, b and c show the karyotypes of Bornean sambar deer, rusa deer and $F_{1}$ hybrid, respectively. All except three of the autosomes of the hybrids were unpaired. 


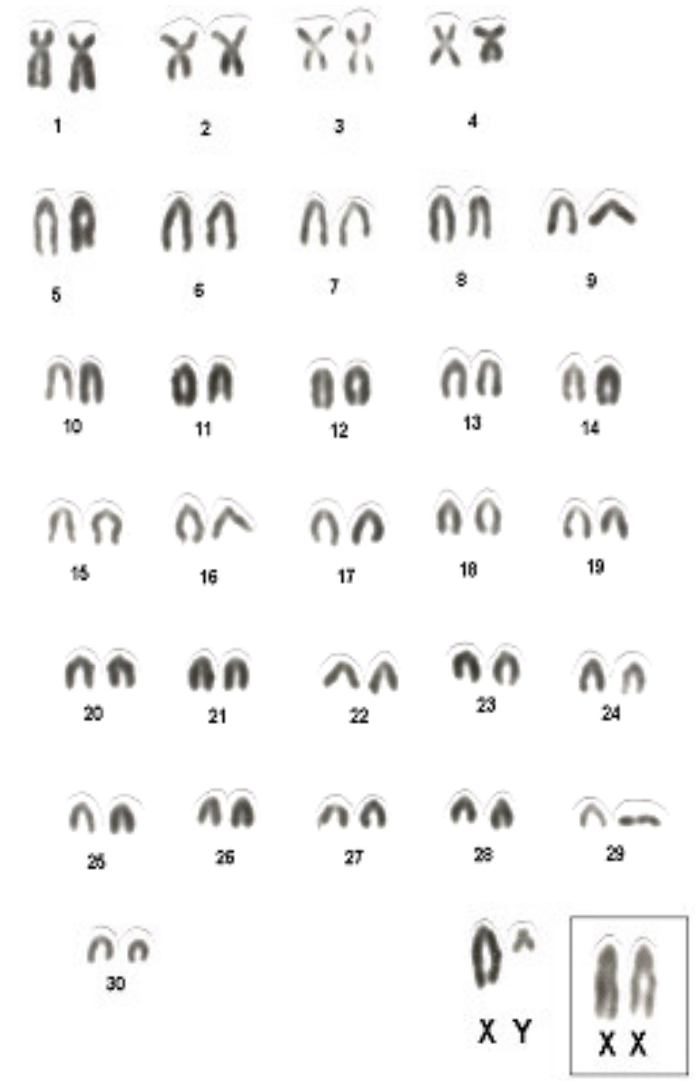

Fig. 1a: Karyotype of male and Female (inset) Bornean sambar deer, $2 n=62$

G-banding: In the present study, the G-banded karyotype of the rusa deer showed that the metacentric and submetacentric chromosomes displayed poor affinity to stain in the centromeric region. The large chromosomes and $\mathrm{X}$ chromosome showed a characteristic G-banding pattern providing a correct identification of the homologs. The small chromosomes showed some G-bands. In these chromosomes only one or two bands were present. The chromosomes were arranged according to Reading Conference nomenclature ${ }^{[10]}$, by matching the G-banding patterns of the two parental species, the equivalent pairs of autosomal chromosomes of the Bornean sambar deer (Fig. 2a) have been arranged as close as those of the rusa deer (Fig. 2b).

The G-banded karyotype of the male $F_{1}$ hybrid is shown in Fig. 2c. The chromosomes were arranged to show the similarity with those of the parental species. One unpaired large submetacentric autosome of the $F_{1}$ hybrid clearly comes from the rusa and the other two unpaired acrocentrics come from the Bornean sambar deer.

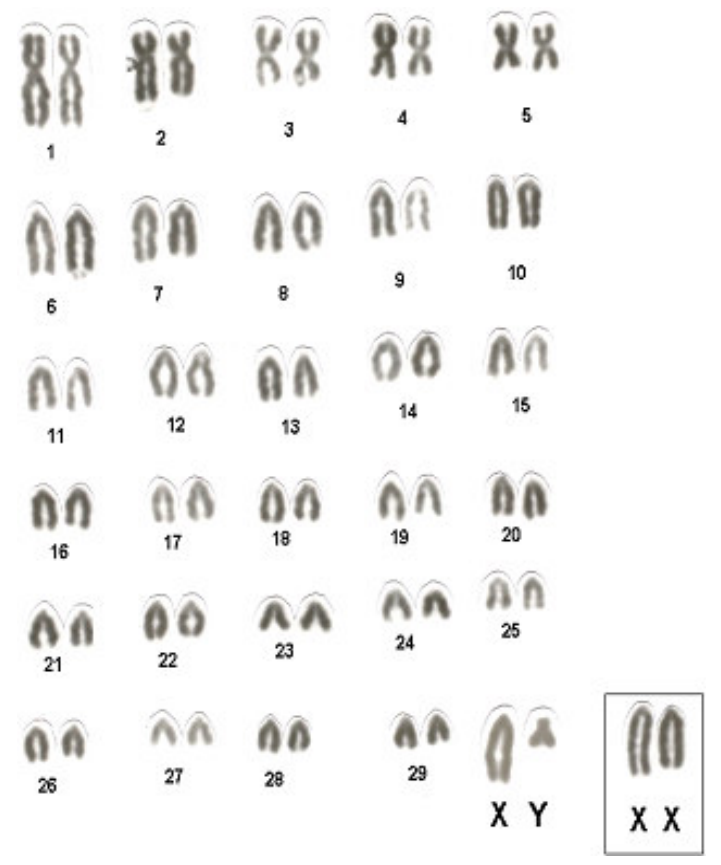

Fig. 1b: Karyotype of male and Female (inset) Rusa deer, $2 n=60$

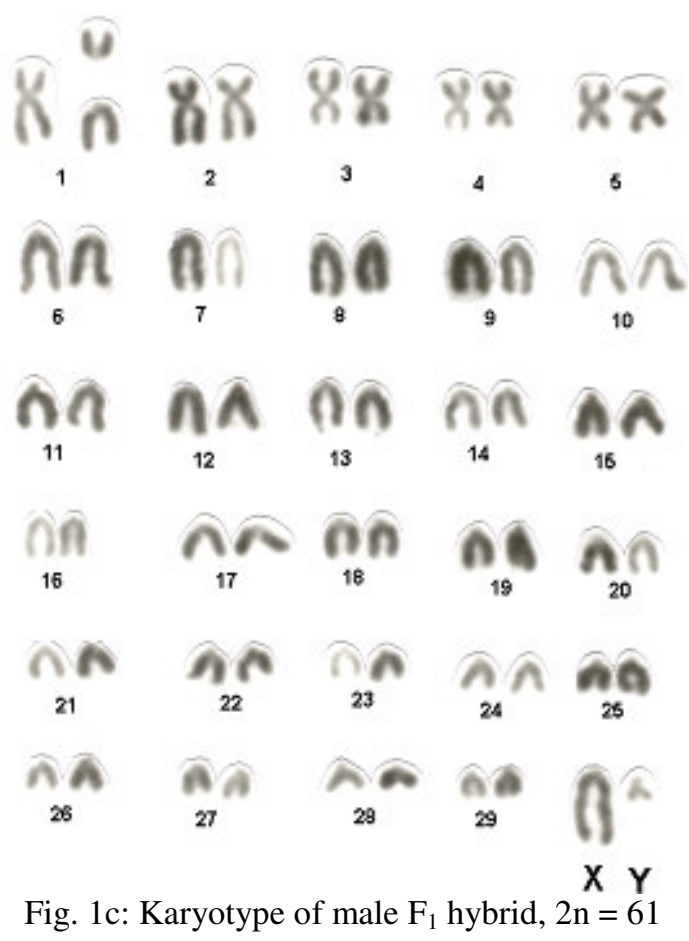

Nucleolar organizer regions: The first statistical analysis concerned the total amount of positively stained NORs for each nucleolar chromosomes in each animal. 


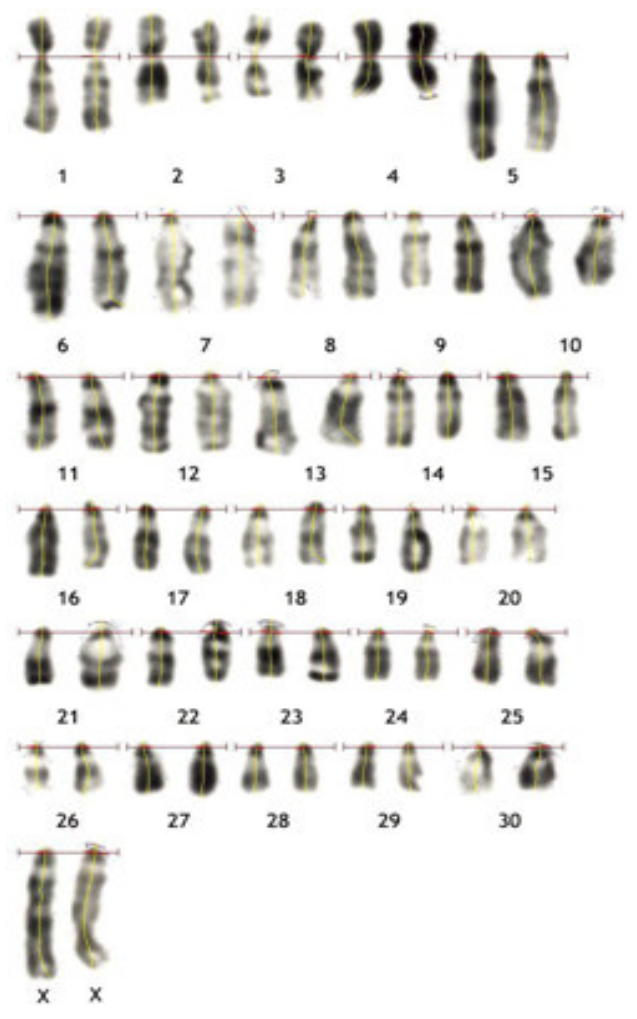

Fig. 2a: G-banded Karyotype of Bornean sambar deer

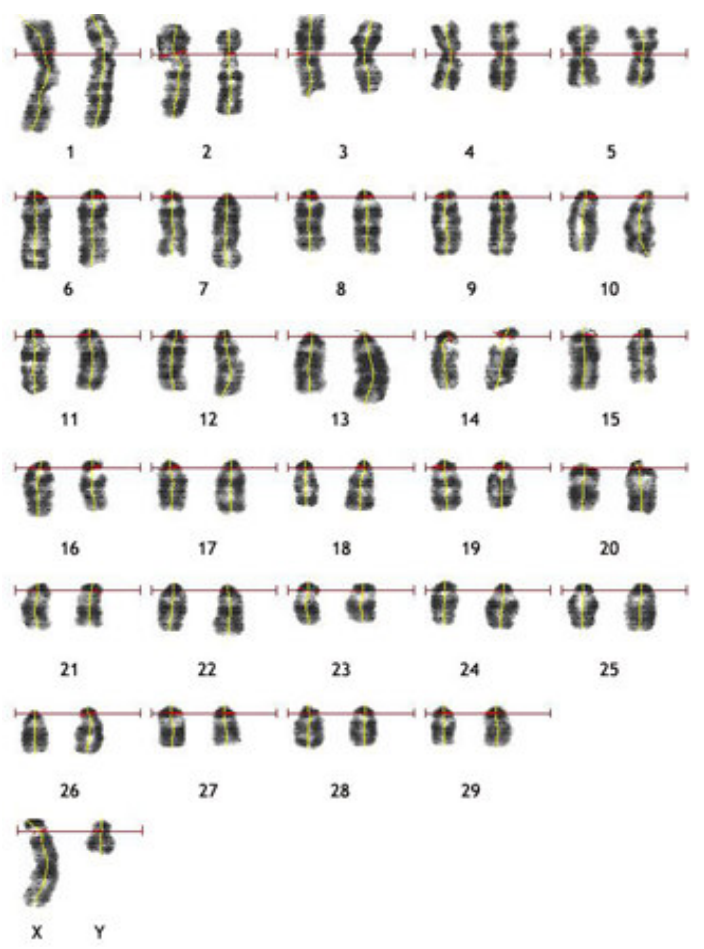

Fig. 2b: G-banded Karyotype of rusa deer

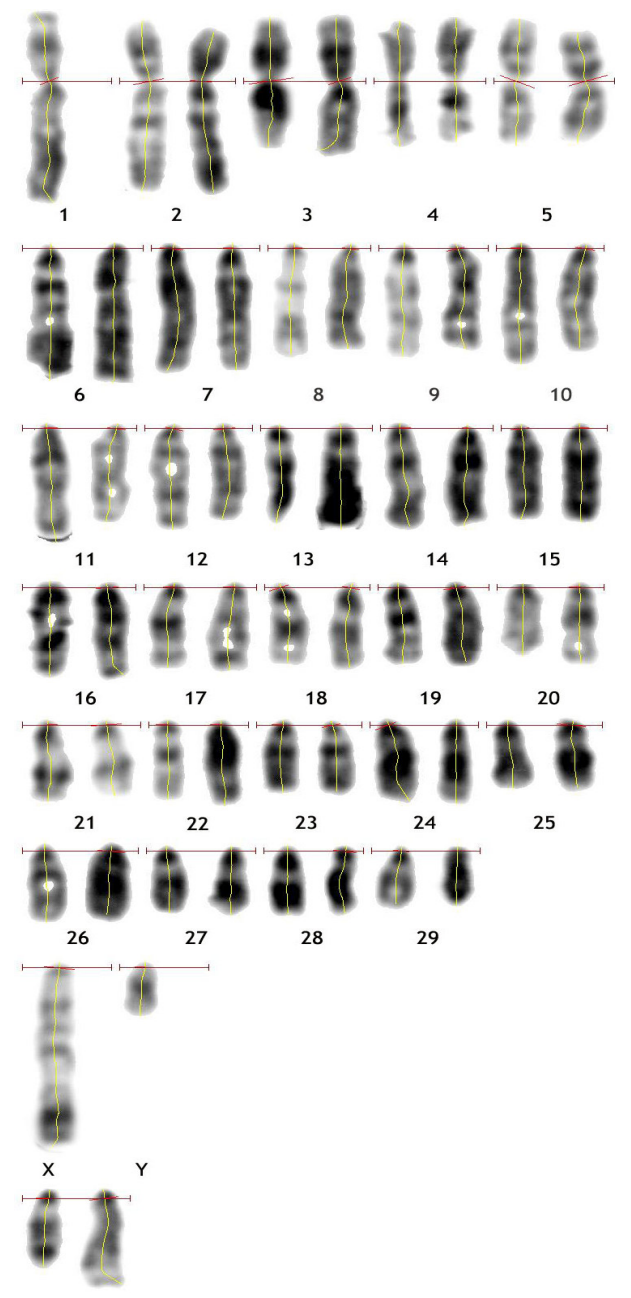

Fig. 2c: G-banded Karyotype of $F_{1}$ hybrids

The number of nucleolar organizer regions (NORs) band varied in the cells of parental species and their $F_{1}$ hybrids. Both parental species were always observed as Ag-NOR positive, the NORs located at the telomeric sites of two different chromosomes of both parental species. In metaphase cells of male and female Bornean sambar deer, the NORs were detected on a single homologue of the metacentric chromosome 5 and on the acrocentric chromosome 7 (Fig. 3a). Male rusa deer displayed NORs on both homologous chromosomes 1 and 6 (Fig. 3b). The female rusa deer appeared to have NORs on one homologous chromosome 1 and both homologue of chromosome 6 (Fig. 3c). The NORbanded metaphase chromosomes showed variation in the chromosome location in the two types of F1 hybrids (rusa deer $\delta \mathrm{x}$ Bornean sambar deer $q$ and Bornean sambar deer $\delta^{\lambda} \mathrm{x}$ rusa deer ?). 
a

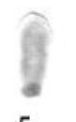

5

b

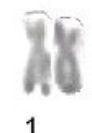

c

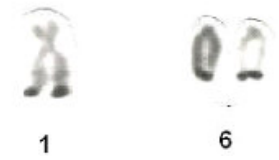

d

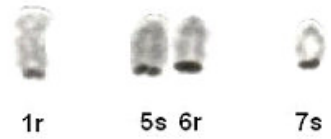

e

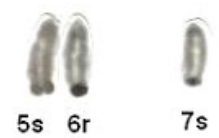

Fig. 3: NORs location of (a): Male Bornean sambar (the female showed the same pattern) (b): Male rusa (c): Female rusa (d): Rusa, $\mathrm{r} \hat{\delta} \mathrm{x}$ Bornean sambar, $\mathrm{s}+\mathrm{F}_{1}$ hybrid and (e): Bornean sambar, $\mathrm{s} \hat{\mathrm{O}} \mathrm{x}$ rusa, $\mathrm{r}+\mathrm{F}_{1}$ hybrid. The parental species for the hybrids were represented by $\mathrm{s}$ and $\mathrm{r}$ for Bornean sambar and rusa, respectively

The Bornean sambar deer $\hat{\partial} \mathrm{x}$ rusa deer $q$ hybrids had NORs terminally located on the chromosome pair 6 (the inherited chromosome 5 of Bornean sambar and chromosome 6 of rusa) and on a single member of homologous pair 7 (from Bornean sambar deer) (Fig. 3e). The rusa $\delta$ x Bornean sambar $q$ hybrid, in addition to having NORs on the chromosome pair 6 and the single homologue of chromosome 7 , also displayed NORs at the telomeric region of the largest metacentric chromosome (Fig. 3d). The parental species for the hybrids in Fig. 3d and e were represented by $\mathrm{s}$ and $\mathrm{r}$ for Bornean sambar and rusa, respectively.

\section{DISCUSSION}

The chromosome and fundamental number found in the Bornean Sambar differed from that previously reported in other Sambar deer species ${ }^{[4]}$. However, for rusa deer they were identical to those previously reported $^{[11]}$. $F_{1}$ hybrids were produced by breeding the Bornean sambar deer with rusa deer. The hybrid karyotype of $2 n=61$ is exactly intermediate between that of the parent species. The hybrid has clearly received four metacentric chromosomes from Bornean sambar deer and five metacentric chromosomes from rusa deer parent. Thus the $F_{1}$ hybrids had a single submetacentric chromosome necessarily from the rusa deer and two unpaired chromosomes from Bornean sambar deer. The advantage of chromosomal

investigation of interspecies hybrids is that haploid chromosomes from each parental species in one metaphase allow consistency in chromosome morphology and banding patterns. Thus the results are more comparable than that from two separate metaphases of the parental species for determining the genetic similarities between Bornean sambar deer and rusa deer.

Previous study ${ }^{[12]}$ indicated a close relationship between sambar deer and rusa deer using protein marker. These markers confirmed a significant level of similarity among the two species although genetic variants for several loci present in some sambar deer were not found in rusa deer. Following this, the relationship in the Cervus species has been elucidated using sequences from complete mitochondrial DNA control regions ${ }^{[13]}$. Their data suggested that the two species were genetically close to each other. The relationships between the two species were further studied to analyze the morphometrical relationship on 15 species of South East Asian deer ${ }^{[14]}$. They suggested that the elements of skull shape as determined by morphometry could depict phylogenetic relationship among the subfamily. Their data indicate that sambar deer and rusa deer have risen from the same root.

In the present study, G-banded karyotype of the two parental species and $F_{1}$ hybrids were compared to show their close relationship based on chromosome bands. The G-band patterns enable all of the homologues to be distinguished despite their gradual decrease in size. Analysis of G-banded karyotype from the parental species has allowed a more accurate assessment of the level of homology between their chromosomes and the determination of type of karyotypic differences. As shown in Fig. 2a and b, Bornean sambar deer and rusa deer have homologous G-banding patterns. Twenty eight pairs of the Bornean sambar autosomes and sex chromosomes shared homology with 28 pairs of autosomes and sex chromosomes of the rusa deer with respect to the patterns of the G-banded chromosomes. Their chromosome complement differs because the rusa deer carries one pair of submetacentric autosome (pair 1) while Bornean sambar deer has two pairs of acrocentric chromosome. Pair 1 of the rusa karyotype is significantly different in size and banding patterns from that of Bornean sambar deer. However in the hybrid karyotype this chromosome is a single pair in the complement.

In mammals, the number of structural NORs per karyotype and their chromosomal distribution are considered to be species-specific ${ }^{[15]}$. However, NOR 
transcriptional activity is very variable and it has been postulated that this is a characteristic of each individual for many mammalian species ${ }^{[16-18]}$. This variability would depend on specific metabolic cell demand and it has been correlated with different cell types ${ }^{[19]}$. In the Cervus species studied, although the location of NOR active site were different, the ribosomal RNA dispersed equally in the Bornean sambar deer and rusa deer, with 3 active ribosomal sites. Besides this, the male and female rusa deer displayed active NORs at different locations. The differences between the Bornean sambar deer and rusa deer with regards to the number and location of active NORs indicate that there has been loss, fusion or splitting of the regions in the karyotype evolution of these animals.

Interspecies hybrids have often shown that ribosomal genes of one parental species are transcriptionally dominant over those of the other species. In vertebrate, nuclear dominance has been detected in hybrids between amphibian species ${ }^{[20]}$ and hybrid between horse and ass ${ }^{[17]}$. However, in this study, it was found that the activity of both parental ribosomal genes function codominantly in the hybrids although only the rusa deer $\delta \mathrm{x}$ Bornean sambar deer $q$ hybrid showed NOR activity on chromosome 1 of rusa deer. The two parental homologous chromosomes frequently express their ribosomal genes when both carry structural NORs (pair 6). Nevertheless, it was always the case that only one of the homologous chromosome 7 showed active NORs in most of the hybrids. This NOR activity would necessarily correspond to the expression of sambar rRNA genes, since chromosome 7 of rusa does not carry structural NORs. The hybrids appeared to have active NORs originating from both parents considering the chromosome pair 5 and 7 of the Bornean sambar deer had identical morphology and G-band pattern, with chromosome pair 6 and 8 of the rusa, respectively.

The present study reports hybrids with uneven chromosome number. Studies on hybrids with uneven chromosome number in buffaloes ${ }^{[21]}$ showed that the hybrids were fully fertile. However, the reduction in fertility in cattle hybrid with $2 n=59$ were observed resulting from a non-equivalence of the homologous segments of chromosomes and by a gene diversity in the heterozygous $\operatorname{sex}^{[22]}$. He reported that the male hybrid was sterile while the female was fertile. In this present study, the interspecific dissimilarities with regards to chromosome number and morphology are less extensive and the production of chromosomally balanced gametes could be expected.

\section{CONCLUSION}

Cytogenetic analysis of the two subspecies of deer and their crossbreds, obtained through natural mating, allowed the identification of G-banding and NORs locations. This result confirms the effectiveness of hybridization process between two different species of deer. Moreover, the presence of ribosomal genes at different location of the hybrids might be used as a chromosome marker in breeding purposes. In the present study, natural mating between Sambar deer and Rusa deer were conducted and the number and chromosomal location of the nucleolar organizer regions in their offsprings were analyzed by the silver staining method. Apart from that, the interspecific dissimilarities with regards to chromosome number and morphology are less extensive and the production of chromosomally balance gametes could be expected.

\section{ACKNOWLEDGEMENT}

The researchers wish to express their sincere thanks to the field staff of the Livestock Breeding Station, Sabrang, Keningau, Sabah, in East Malaysia, under the leadership of the station manager, Mr. Alexius Jalani, for their assistance in the management of the animals and field gathering of data. This study was supported by IRPA grant 01-02-04-0038-EA001 to Universiti Putra Malaysia from the Ministry of Science, Technology and the Environment, Malaysia.

\section{REFERENCES}

1. Gustavsson, I., M. Switonski, L. Larason and L. Ploen, 1998. Synaptonemal complex analysis of spermatocytes in hybrids of silver fox and blue fox. J. Hered., 79: 338-343. http://jhered. oxfordjournals.org/cgi/content/abstract/79/5/338.

2. Winter, H., B. Mayr, W. Schleger, E. Dworak, J. Krutzler and M. Kalat, 1986. Genetic characterization of the mithun (Bos frontalis) and studies of spermatogenesis, blood groups and haemoglobins of its hybrids with Bos indicus. Res. Vet. Sci., 40: 8-17. http://www.ncbi. nlm.nih.gov/pubmed/3704329.

3. Neusser, M., M. Munch, G. Anzenberger and S. Muller, 2005. Investigation of marmoset hybrids (Cebuella pygmaea $\mathrm{x}$ Callithrix jacchus) and related Callitrichinae (Pltyrrhini) by cross-species chromosome painting and comparative genomic hybridization. Cytogenet. Genome Res., 108: 191-196. DOI: 10.1159/000080815. 
4. Nugent, G., J.P. Parkes and K.G. Tustin, 1987. Changes in the density and distribution of red deer and wapiti in Northern Fiordland. N. Z. J. Ecol. 10: 11-21. http://cat.inist.fr/ ?aModele $=$ afficheN\&cpsidt $=7104584$.

5. Muir, P.D., G. Semiadi, G.W. Asher, T.E. Broad, M.L. Tate and T.N. Barry, 1997. Sambar deer (C. unicolor) $\mathrm{x}$ Red deer (C. elaphus) interspecies hybrids. J. Hered., 88: 366-372. http://jhered. oxfordjournals.org/cgi/reprint/88/5/366.

6. Davidson, M.N., 1983. Sika Deer. In: The Handbook of New Zealand Mammals, King, C.M., (Ed.). Oxford University Press, USA., pp: 468-477.

7. Ismail, I., M.M. Norbazlin and M. Saidi, 2004. Gbanded karyotype constitutive heterochromatin and NORs in the Rusa deer. Proceedings of the 11th Animal Science Congress of the AsianAustralasian Association of Animal Production Societies, Sep. 5-9, Kuala Lumpur, Malaysia.

8. Rubini, M. and F. Fontana, 1988. Standard Gbanded karyotype, constitutive heterochromatin and nucleolus organizer regions in the roe deer (Capreolus capreolus, L.). Genetica, 77: 143-148. http://cat.inist.fr/?aModele $=$ afficheN\&cpsidt $=731$ 6895.

9. Cao, X., H. Jiang and X. Zhang, 2005. Polymorphic karyotypes and sex chromosomes in the tufted deer (Elaphodus cephalophus): Cytogenetic studies and analyses of sex chromosome-linked genes. Cytogenet Genome Res., 109: 512-518. DOI: 10.1159/000084212.

10. ISCN., 1978. An international system for cytogenetic nomenclature. Cytogenet. Cell. Genet., 21: 309-404. DOI: 10.1159/130909.

11. Wang, S. and R. Du, 1988. Karyotype of cervidae and their evolution. Acta Zool. Sin, 29: 214-222. http://www.actazool.org/searchresult.asp?fulltext= karyotype+of+cervidae.

12. Emerson, B.C. and M.L. Tate, 1993. Genetic analysis of evolutionary relationship among deer (subfamily Cervinae). J. Hered., 84: 266-273. http:// jhered.oxfordjournals.org/cgi/reprint/84/4/266.

13. Randi, E., N. Mucci, F. Claro-Hergueta, A. Bonnet and E.J.P. Douzery, 2001. A mitochondrial DNA control region phylogeny of the Cervinae: Speciation in Cervus and implications for conservation. Anim. Conserv., 4: 1-11. DOI: 10.1017/S1367943001001019.
14. Meijaard, E. and P. Groves, 2004. Morphological relationship between South-East Asian deer (Cervidae, tribe Cervini): Evolutionary and biogeographic implication. J. Zool. Lond., 263: 179-196. DOI: 10.1017/S0952836904005011.

15. Walker, J.L., M. Rojas, S. Flores, A. Spotorno and G. Manriquez, 1999. Genomic compatibility between two phyllotine rodent species evaluated through their hybrids. Hereditas, 131: 227-238. DOI: 10.1111/j.1601-5223.1999.00227.x.

16. Makinen, A. and I. Gustavsson, 1982. A comparative chromosome banding study in the silver fox, blue fox and their hybrids. Hereditas, 97: 289-297. http://www.fao.org/agris/ search/display.do?f=./1983/v907/SE8310404.xml; SE8310404.

17. Kopp, E., B. Mayr, M. Kalat and W. Schleger, 1988. Polymorphisms of NORs and heterochromatin in the horse and donkey. J. Hered., 79: 332-337. http://jhered.oxfordjournals.org/ cgi/reprint/79/5/332.

18. Mellink, C.H.M., A.A. Bosma, N.A. de Haan and A.A. McDonald, 1992. Numerical variation of nucleolar organizer regions after silver staining in domestic and wild suidae (mammalia). Anim. Genet., 23: 231-239. http://www.ncbi. nlm.nih.gov/pubmed/1503258.

19. Berrios, S., J. Koifman and R. Fernandez-Donoso, 1992. Tissue and sex differences in the expression of nucleoli in mouse somatic cells. Eur. J. Morphol., 30: 297-303. http://www.ncbi. nlm.nih.gov/pubmed/1305830.

20. Cassidy, D.M. and A.W. Blacker, 1974. Repression of nucleolar organizer activity in an interspecific hybrid of the genus Xenopus. Develop. Biol., 41: $\quad 84-96 . \quad$ http://www.ncbi.nlm. nih.gov/pubmed/4435304.

21. Bongso, T.A. and M. Hilmi, 1982. Chromosome banding homologies of tandem fusion in river, swamp and crossbred buffaloes (Bubalus bubalis). Can. J.Genet. Cytol., 24: 667-673.

22. Popescu, C.P., 1969. Ideograms of Yak (Bos grunniens), cattle (Bos Taurus) and their hybrid. Ann. Genet. Sel. Anim., 1: 207-217. http://www. gse-journal.org/index.php?option=article\&access =standard\&Itemid=129\&url=/articles/gse/pdf/1969/ 03/AGSE_0003-4002_1969_1_3_ART0001.pdf. 